\title{
A Case of Complicated Prosthetic Valve Endocarditis Successfully Managed Conservatively
}

\author{
Sanjana Shah, $\mathrm{MBBS}^{1^{*}}$ and Abhisheik Prashar, MBBS, MMed ${ }^{2}$ \\ ${ }^{1}$ St. George Hospital, Australia \\ ${ }^{2}$ Department of Cardiology, St. George Hospital, Australia
}

\begin{abstract}
Prosthetic valve endocarditis (PVE) is associated with high morbidity and carries a guarded overall prognosis. The management of this condition is based on consensus guidelines determined by various high-risk clinical and radiological features. Early surgery along with antibiotics has been the preferred option conventionally. We present a case of PVE with high-risk features that was successfully managed with antibiotics. This highlights the need for individualizing treatment based on prognostic evaluation rather than subjecting all patients to high-risk cardiac surgery.
\end{abstract}

Keywords

Prosthetic valve endocarditis, Infective endocarditis, Vegetation

\section{Introduction}

Prosthetic valve endocarditis (PVE) can affect both bio-prosthetic and mechanical heart valves. It carries an incidence rate of 0.3 to $1.2 \%$ patient-year $[1,2]$. The general approach to the management of these conditions is using a combination of medical and surgical modalities of treatment. Valve surgery is inherently high-risk and there is limited randomised data examining the role of early valve surgery. Thus, there remains contention as to the best management of such patients. We report a case of a patient who presented with prosthetic aortic valve endocarditis complicated by complete heart block, successfully managed conservatively, without aortic valve replacement.

\section{Case Presentation}

A 68-year-old woman presented to the emergency department with four episodes of dizziness in the preceding 48 hours. She had one syncopal event during this time. She also complained of intermittent palpitations and rigors. Further history revealed that she had recurrent intermittent pre-syncopal events without loss of consciousness for several weeks. She lost six-kilograms of weight during this time. In the weeks prior to presentation, she had been treated by her local doctor with two courses of oral cephalexin for suspected urinary tract infection, and another course of oral roxithromycin for suspected sinusitis. She underwent a dental procedure a month before this presentation without antibiotic cover. Her past medical history included a bioprosthetic aortic valve replacement performed five years ago, for severe aortic stenos is secondary to a bicuspid aortic valve.
On examination, she had a low-grade temperature of 37.8 degrees C, a blood pressure of $110 / 60 \mathrm{mmHg}$ and a heart rate of 50 beats per minute. There were no peripheral stigmata of infective endocarditis. Her cardiovascular examination was normal with dual heart sounds and no murmurs on auscultation. Her baseline bloods showed an elevated white cell count of $15.710^{\wedge} \mathrm{g} / \mathrm{L}$ and a c-reactive protein (CRP) of 71 $\mathrm{mg} / \mathrm{L}$. All other blood tests including liver function tests, thyroid function tests, electrolytes and creatinine were normal. A chest X-ray did not show any focus of consolidation and the urine microscopy and culture did not grow any microorganisms. Electrocardiogram was performed which demonstrated a complete heart block. In view of her fever, new complete heart block and a history of aortic valve replacement, infective endocarditis was suspected. Blood cultures were collected and a transoesophageal echocardiogram (TOE) was performed to look for evidence of infective endocarditis. A mobile, echodense lesion measuring $10 \mathrm{~mm} \times 7 \mathrm{~mm}$ in diameter was noted on the cusp of the prosthetic aortic valve, with an associated area of oedema adjacent to the aortic root (Figure 1). Howev-

*Corresponding author: Sanjana Shah, St. George Hospital, Gray Street, Kogarah, NSW 2217, Australia, Tel: +61-415411207, Fax: +6191133992

Accepted: February 20, 2021

Published online: February 22, 2021

Citation: Shah S, Prashar A (2021) A Case of Complicated Prosthetic Valve Endocarditis Successfully Managed Conservatively. Ann Heart 5(1):110-113 


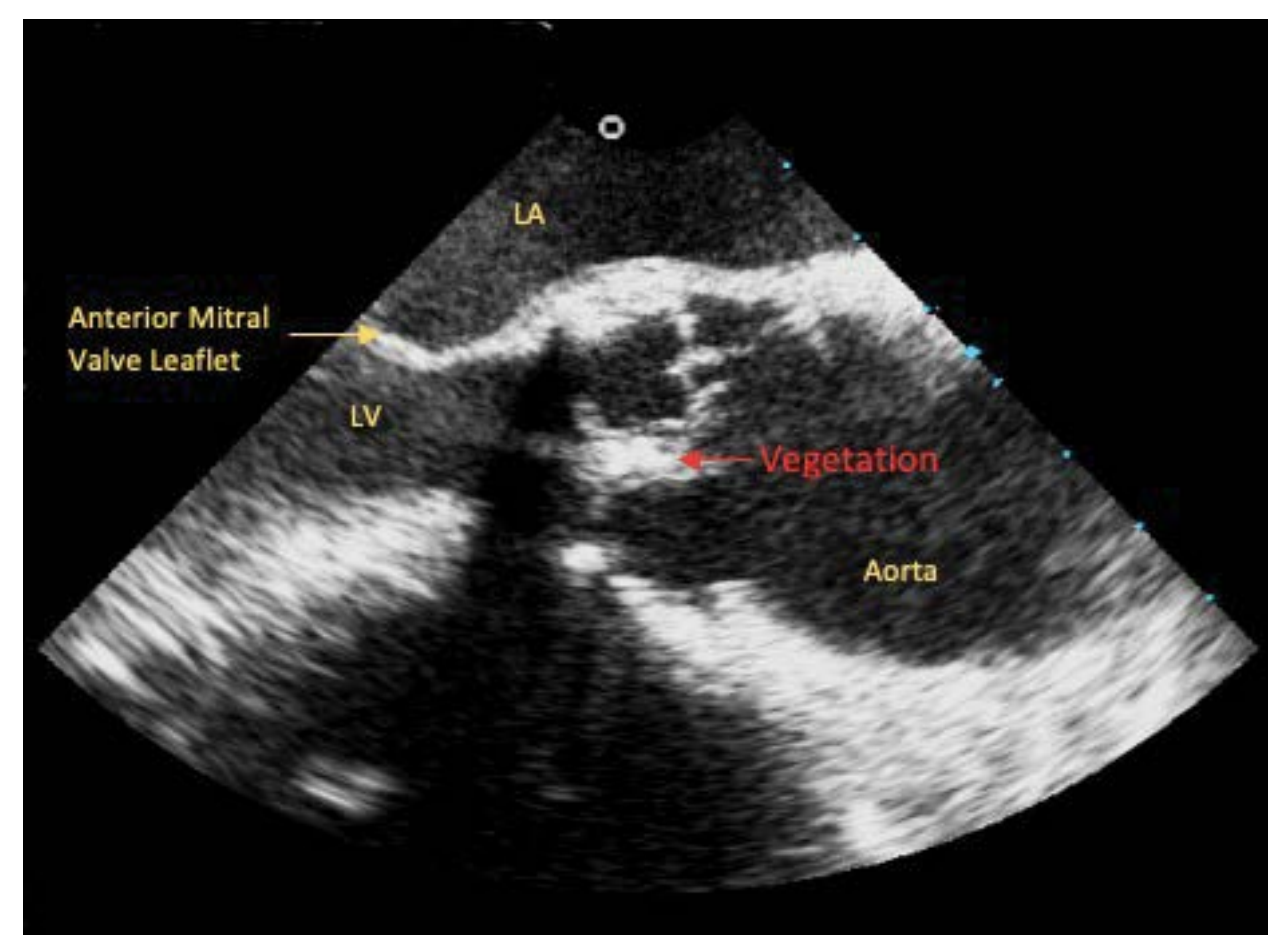

Figure 1: Trans-oesophageal echocardiogram - 3 chamber view $10 \mathrm{~mm} \times 7 \mathrm{~mm}$ vegetation (red arrow) on the bio-prosthetic aortic valve. LA: Left atrium; LV: Left ventricle

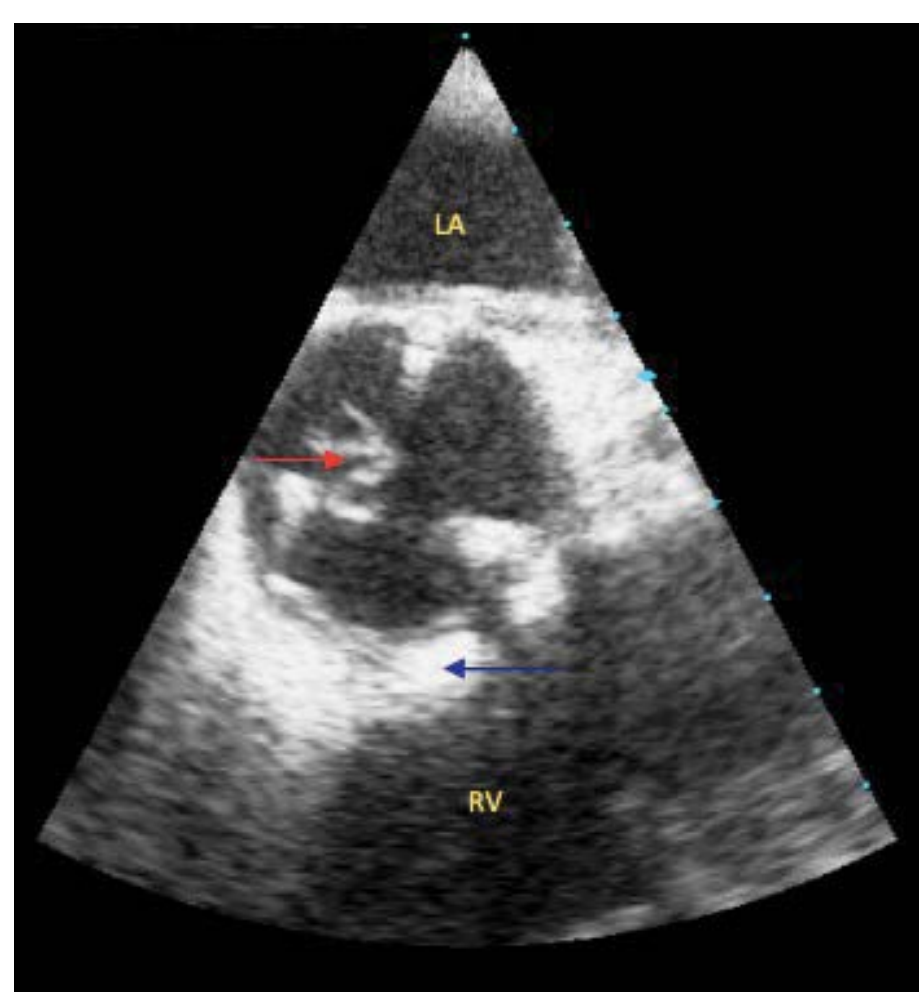

Figure 2: Trans-oesophageal echocardiogram - short-axis view $10 \mathrm{~mm} \times 7 \mathrm{~mm}$ vegetation (red arrow) seen on the bioprosthetic aortic valve with no evidence of aortic root abscess (blue arrow).

LA: Left atrium; RV: Right ventricle.

er, no annular or aortic root abscesses were noted on the echo (Figure 2). Five sets of blood cultures performed in the first few days of hospital stay were all negative.
The patient was commenced on intravenous antibiotic therapy with a combination of ceftriaxone, vancomycin and gentamicin for one week following which the ceftriaxone was 
ceased based on recommendations made by the infectious diseases team. Although hemodynamically stable, she remained in complete heart block with an accelerated ventricular rate of 55-60 beats per minute. In the context of concurrent infection, decision was made against insertion of temporary pacing wires to minimize the risk of further dissemination of infection. She developed pauses, up to 4.9 seconds and was commenced on an isoprenaline infusion. Repeated attempts at weaning isoprenaline were unsuccessful due to prolonged pauses. She was reviewed by the cardiothoracic team with an intention to undergo an aortic valve replacement. However, her CRP had normalised, she was afebrile, and there was no further clinical deterioration. Progress TOE revealed that the vegetations and aortic regurgitation noted on the first study were regressing and CT scan of the chest revealed no evidence of embolization. The risks and benefits of surgical intervention were discussed with the patient, who declined surgery and preferred to continue trial of medical management. She continued on intravenous antibiotic therapy for a total duration of six weeks. A permanent pacemaker was inserted after five weeks of antibiotic therapy, in the context of sustained period of apyrexia and normal white cell counts. A repeat TOE was performed on completion of treatment that showed no evidence of vegetations. The patient was discharged home, and remained well on subsequent outpatient follow up at 6 weeks with no further clinical or echocardiographic features of endocarditis.

\section{Discussion}

Infective endocarditis is a serious and life-threatening complication in patients with prosthetic valves [3]. The weighted incidence for infections of bio-prostheses calculated from published series is $0.91 \%$ for aortic valves, with infections primarily restricted to the cusps, resulting in bio prosthetic failure [1]. The in-hospital mortality risk associated with it ranges between 20 to $80 \%$ [3].

Traditionally, early valve surgery has been central to the standard of care for treating patients with PVE. This practice is mostly based on expert opinion and limited observational studies [4]. More recently, the role of surgery in therapy of PVE has been questioned with some studies suggesting benefit [5], some suggesting harm [6], whilst others showing no statistically significant association between valve surgery and outcome [7]. Operative in hospital mortality has remained high, over the last few decades. In a large, multi-national prospective, cohort study, no mortality benefit was noted with early valve surgery after adjusting for treatment, survival and selection bias in patients with PVE [8]. Also, there is a scarcity of reports on the long-term follow-up of patients with well-defined endocarditis [9].

Current American Heart Association guidelines recommend early surgical management in the presence of complicated PVE. This includes concomitant heart failure, severe valve dysfunction and dehiscence, locally uncontrolled infection, lesion caused by fungi or other highly resistant organisms and persistent bacteraemia despite appropriate anti-microbial therapy for 5-7 days after ruling out other sites of infection [10]. The European Society of Cardiology have also released similar guidelines for urgent surgical intervention which in addition to the above criteria also include the persistence of large vegetations (>10 $\mathrm{mm}$ ) despite appropriate anti-microbial treatment or those associated with valve stenos is or regurgitation [11].

Some special aspects need to be considered in the medical management of PVE. It usually results in larger vegetations than those seen with native valve infections. As a result, longer duration of antibiotics is required in higher, non-toxic doses to penetrate the vegetations entirely [1]. Predicting likely success of non-surgical treatment of infective endocarditis relies on the use of clinical and biochemical parameters to stratify risk of complications. Some studies have suggested persistent infection as a sign of failure of conservative management [12], and are associated with a high mortality [13]. In addition, an early case series suggests diabetes mellitus to be an independent predictor of mortality [14].

Our patient demonstrated some features which would be considered high risk-diabetes, complete heart block suggesting bioprosthetic ring infection. This case highlights a case of prosthetic valve endocarditis with high risk features, which resolved completely with antibiotics alone.

To the best of our knowledge, no prospective randomized-controlled trials have been performed to support early surgical intervention in patients with PVE. There are also no specific guidelines for the monitoring of patients who have been managed with antibiotics alone. This conservative approach may be used in some patients with PVE who show clinical and biochemical improvement with antibiotics. Treatment should be individualized, with a clear plan of intervention, close monitoring for detection of complicating factors, and subsequent plan of action.

\section{Disclosure}

The authors have no relationships with the industry, financial associations or conflicts of interest to disclose.

\section{References}

1. Piper C, Korfer R, Horstkotte D (2001) Prosthetic Valve Endocarditis. Heart 85: 590-593.

2. Wang A, Athan E, Pappas PA, et al. (2007) Contemporary clinical profile and outcome of prosthetic valve endocarditis. JAMA 297: 1354-1361.

3. Habib G, Thuny F, Avierinos JF (2008) Prosthetic valve endocarditis: current approach and therapeutic options. Prog Cardiovasc Dis 50: $274-281$.

4. Attaran S, Chukwuemeka A, Punjabi PP, et al. (2012) Do all patients with prosthetic valve endocarditis need surgery? Interact Cardiovasc Thorac Surg 15: 1057-1061.

5. Aksoy O, Sexton DJ, Wang A, et al. (2007) Early surgery in patients with infective endocarditis: a propensity score analysis. Clin Infect Dis 44: 364-372.

6. Tleyjeh IM, Kashour R, Zimmerman V, et al. (2008) The role of valve surgery in infective endocarditis management: A systematic review of observational studies that included propensity score analysis. Am Heart J 156: 901-909. 
7. Wang A, Pappas P, Anstrom KH, et al. (2005) The use and effect of surgical therapy for prosthetic valve infective endocarditis: $A$ propensity analysis of a multicenter, international cohort. Am Heart J 150: 1086-1091.

8. Lalani T, Chu VH, Park LP, et al. (2013) In-hospital and 1-year mortality in patients undergoing early surgery for prosthetic valve endocarditis. JAMA Intern Med 173: 1495-1504.

9. Olaison L, Hogevik P, Myken P, et al. (1996) Early surgery in infective endocarditis. QJM 89: 267-278.

10. Baddour LM, Wilson WR, Bayer AS, et al. (2015) Infective endocarditis in adults: Diagnosis, antimicrobial therapy, and management of complications: a scientific statement for healthcare professionals from the American Heart Association. Circulation 132: 1435-1486.
11. Habib G, Lancellotti P, Antunes MJ, et al. (2015) 2015 ESC guidelines for the management of infective endocarditis: The task force for the management of infective endocarditis of the European Society of Cardiology (ESC) endorsed by: European Association for Cardio-Thoracic Surgery (EACTS), the European Association of Nuclear Medicine (EANM). Eur Heart J 36: 3075-3128.

12. Hogevik H, Olaison L Andersson R, et al. (1995) Epidemiologic aspects of infective endocarditis in an urban population. A 5-year prospective study. Medicine 74: 324-339.

13. Tingleff J, Egeblad H, Gotzsche CO, et al. (1995) Perivalvular cavities in endocarditis: Abscesses versus pseudoaneurysms? A transesophageal Doppler echocardiographic study in 118 patients with endocarditis. American Heart Journal 130: 93-100.

14. Chu VH, Cabell CH, Benjamin DK, et al. (2004) Early predictors of in-hospital death in infective endocarditis. Circulation 109: 1745-1749. 